\title{
Perturbations of the solar wind flow by radial and latitudinal pick-up ion pressure gradients
}

\author{
H. J. Fahr and K. Scherer \\ Institut für Astrophysik und extraterrestrsiche Forschung, Universität Bonn, Germany
}

Received: 12 June 2003 - Revised: 19 September 2003 - Accepted: 1 December 2003 - Published: 14 June 2004

\begin{abstract}
It has been found that pick-up ions at their dynamical incorporation into the solar wind modify the original conditions of the asymptotic solar wind plasma flow. In this respect, it has meanwhile been revealed in many papers that these type of solar wind modifications, i.e. deceleration and decrease of effective Mach number, are not only due to the pick-up ion loading effects, but also to the action of pick-up ion pressure gradients. Up to now only the effects of radial pick-up ion pressure gradients were considered, however, analogously but latitudinal pressure gradients also appear to be important. Here we study the effects of radial and latitudinal pick-up ion pressure gradients, occurring especially during solar minimum conditions at mid-latitude regions where slow solar wind streams change to fast solar wind streams. First, we give estimates of the latitudinal wind components connected with these gradients, and then after revealing its importance, present a more quantitative calculation of solar wind velocity and density perturbations resulting from these pressure forces. It is shown that the relative density perturbations near and in the ecliptic increase with radial distance and thus may well explain the measured non-spherically symmetric density decrease with distance. We also show that the solar wind decelerations actually seen with Voyager-1/2 are in conciliation with interstellar hydrogen densities of $n_{H_{\infty}} \geq 0.1 \mathrm{~cm}^{-3}$, in contrast to earlier claims for $n_{H_{\infty}}=0.05 \mathrm{~cm}^{-3}$.
\end{abstract}

Key words. Interplanetary physics (solar wind plasma; general and miscellaneous)

\section{Introduction to pick-up ion pressures}

For quite some time it has been recognized that the solar wind dynamics at larger distances is not determined by its own right, but is influenced by the process of incorporating newly created pick-up ions into the solar wind plasma bulk.

Correspondence to: K. Scherer

(kscherer@astro.uni-bonn.de)
We only briefly may consider here this pick-up ion-induced modulation effect. The prediction that the solar wind should become decelerated due to the pick-up ion loading has already been in the literature for many years (see, e.g. Holzer and Leer, 1973; Fahr, 1973; Ripken and Fahr, 1983; Fahr and Ripken, 1984). However, the distant NASA space probes did not yet confirm the expected degree of deceleration (see Richardson et al., 1995). Therefore, more recently, in addition to the ion-loading effect, the dynamical action of the pick-up ion pressure was investigated and it could be shown that by this action, a substantial part of the expected deceleration is compensated (Fahr and Fichtner, 1995; Lee, 1997; Whang, 1998; Wang and Belcher, 1999; Fahr and Rucinski, 1999, 2001).

When becoming decelerated, the solar wind plasma is also heated, both due to adiabatic compression compared to an undecelerated flow and due to being loaded with suprathermal pick-up ions. Thus, the effective solar wind sound speed is increased (see Fahr and Rucinski, 1999, 2001) and the shock compression ratio at the termination shock (TS) is reduced. Connected with these phenomena of deceleration and heating, the solar wind Mach numbers, of course, also decrease with increasing solar distances. The effective Mach number and the wind deceleration are fairly well calculated by making use of an expression for the pick-up pressure, $P_{p u i}=\alpha \rho_{\text {pui }} V_{w}^{2}$, which has been justified in papers by Fahr (2002) and Fahr and Chashei (2002). Here, $\alpha$ represents a weak function of the solar distance $r$, and $\rho_{p u i}$ and $V_{w}$ are the pick-up ion mass density and the solar wind bulk velocity, respectively. The dynamical effect of this pressure $P_{p u i}$ has, however, not yet been taken into account in a fully consistent form, since this would require the simultaneous and consistent treatment of the solar wind plasma, the pick-up ions and the involved MHD waves (see Chashei et al., 2003).

Semi-consistent solutions of a kinetic pick-up ion transport equation prescribing the solar wind velocity profile have, meanwhile, been presented (see, e.g. Chalov et al., 1995, 1997; Fichtner et al., 1997) permitting the calculation of the pick-up ion pressure as a velocity moment of the 
pick-up ion distribution function and supporting a representation of the pick-up pressure in the above mentioned form, i.e. $P_{\text {pui }}=\alpha \rho_{\text {pui }} V_{w}^{2}$. With this expression the decelerated solar wind is described by (see Fahr and Rucinski, 2001):

$$
\frac{d}{d r} V_{w}=\frac{-m_{p} \beta_{e x} \frac{1+\alpha}{\rho_{w}+\rho_{i}}+\frac{2 \alpha}{r} \xi V_{w}}{1+\alpha \xi} .
$$

Here, $\beta_{e x}$ denotes the local injection rate of pick-up ions, which is assumed to be exclusively due to charge exchange processes with protons (photoionization is neglected) and thus is given by $\beta_{e x}=\sigma_{e x} n_{H} n_{w} V_{w}, \sigma_{e x}$, with $n_{H}$ and $n_{w}$ denoting the charge exchange cross sections, with $\sigma_{e x}=2$. $10^{-15} \mathrm{~cm}^{2}$ (McNutt et al., 1999), the local H-atom density and the total proton density, respectively. The function $\xi=\rho_{i} /\left(\rho_{w}+\rho_{i}\right)$ denotes the relative abundance of pick-up ions, with respect to all protons and is adopted here as calculated by Fahr and Rucinski (1999), who used the "hot" kinetic $\mathrm{H}$-atom model developed by Wu and Judge (1979) to describe the $\mathrm{H}$-atom density $n_{H}$. It should be noted here that Eq. (1) contains two important effects: the pick-up ion momentum loading (first term in the numerator of Eq. (1)) and the pick-up ion pressure effect (second term in the numerator of Eq. (1)). The integration of the differential Eq. (1) then yields:

$V_{w}=V_{w 0} \exp \left[\int_{r_{0}}^{r} \frac{\frac{2 \alpha}{r} \xi-n_{H} \sigma_{e x}(1-\xi)(1+\alpha)}{1+\alpha \xi} d r\right]$.

Fahr and Lay (2000) could show that kinetic results of the pick-up ion transport equation can be represented by an algebraic form of the distribution function:

$f_{\text {pui }}=\digamma\left(x^{-0.33}\right) w^{\beta} \exp \left[-C(x)\left(w-w_{0}\right)^{\kappa}\right]$,

where $\digamma$ is a constant, $x=r / r_{0}$ is the radial solar distance in units of $r_{0}=1 \mathrm{AU}, w=\left(v / V_{w}\right)^{2}$ is the squared PUI velocity normalized with $V_{w}$, with $w_{0}$ being a typical injection value. Furthermore, the quantities $\beta, \kappa$, and $C$ are found as: $\beta=-1 / 6 ; \kappa=2 / 3$; and $C(x)=0.442 x^{0.2}$. This distribution function $f_{\text {pui }}$ then leads to the pressure (see Fahr and Rucinski, 2001):

$P_{\text {pui }}=\frac{5}{16} \sqrt[2]{\pi} C(x)^{-3 / 2} \rho_{\text {pui }} V_{w}^{2}=\alpha(x) \rho_{p u i} V_{w}^{2}$.

The function $\alpha$ is obtained by: $\alpha=\alpha(x)=1.83 x^{-0.3}$, meaning that $\alpha$ decreases only slightly with increasing solar distances. It may be noted here that the ratio $P_{\text {pui }} / \rho_{\text {pui }}$ also turned out to be constant in a much earlier and easier approach presented by Vasyliunas and Siscoe (1976). The upper formula (4) is valid at distances $x \geq x_{c}=15$, where $\alpha=\alpha_{c}=\alpha\left(x_{c}\right)$ evaluates to $\alpha_{c}=0.44$. In the following calculations we shall thus assume that $P_{p u i}$ can be represented with sufficient accuracy by Eq. (4) with setting $\alpha(x)=\alpha_{c}$.

After expanding the exponential in Eq. (2) for small arguments one can find that the relative deceleration of the solar wind $\Delta V_{w}=\left(V_{w 0}-V_{w}\right) / V_{w 0}$ is given by:

$$
\Delta V_{w}(x)=\int_{1}^{x} \frac{\frac{2 \alpha_{c}}{r} \xi-n_{H} \sigma_{e x}(1-\xi)\left(1+\alpha_{c}\right)}{1+\alpha_{c} \xi} d x .
$$

Interesting for our context, one derives from the above equation that the quantity $\Delta V_{w}(x)$ always is below 0.1 , with its exact value being dependent on the interstellar $\mathrm{H}$-atom density $n_{H \infty}$ and the solar distance $x$.

In Sect. 2 the latitudinal pressure gradients and their effects to perturb the solar wind flow are estimated, while in Sects. 4 to 5 a quantitative way is shown to derive a set of linearized equations for the latitudinal and the radial velocity components, as well as for the density variation. The solutions of this set of differential equations are discussed in Sect. 6, and finally, the results are discussed in Sect. 7.

\section{Estimated effect of latitudinal PUI pressure gradients}

In the foregoing section we have studied the effect of the radial gradient of the pick-up ion pressure and could show that it partly compensates for the decelerating effect of the pickup ion loading. The question, however, raises itself as to what effect latitudinal gradients of the pressure $P_{p u i}$ might have. To get a first idea of the effects connected with the latitudinal component of $\nabla P_{p u i}$, we shall begin with an approximative description here.

Adopting a volume of original solar wind plasma that initially moves radially outwards with a purely radial, supersonic solar wind velocity $V_{w}$ (i.e. unperturbed velocity), while then a permanent latitudinal force acts on it, one can estimate the upcome of a perturbative latitudinal velocity component $v_{\theta}=v_{\theta}(t)$, with the time $t$, lapsed at the passage of this volume outwards with the primary velocity $V_{w}$. Sitting in a unit volume of plasma, radially comoving with the primary solar wind, the following evolution of a latitudinal solar wind plasma velocity can be estimated:

$\rho_{0} \frac{d v_{\theta}}{d t}=\rho_{0} V_{w} \frac{d v_{\theta}}{d r}=-\left|\nabla P_{p u i}\right|_{\theta}=-\frac{1}{r} \frac{\partial P_{p u i}}{\partial \theta}$.

With Eq. (4) we then find the pressure gradient $\partial P_{p u i} / \partial \theta$ in Eq. (6) using the following expressions:

$$
\begin{array}{ll}
P_{p u i}=\alpha \rho_{\text {pui }} V_{w}^{2} ; & \rho_{\text {pui }}=\rho_{\text {pui }}(r) ; \\
V_{w}=V_{w}(\theta) ; & \rho_{0}=\rho_{0}(r)
\end{array}
$$

and obtain:

$$
\frac{d v_{\theta}}{d r}=-\frac{1}{r V_{w}} \frac{\alpha \rho_{p u i}}{\rho_{0}} \frac{\partial V_{w}^{2}}{\partial \theta}=-2 \frac{\alpha}{r} \xi(r) \frac{\partial V_{w}}{\partial \theta} .
$$

Here, the function $\xi(r)$ denotes the relative abundance of pick-up ions with respect to all ions and is given by Fahr and Rucinski (1999):

$$
\frac{\rho_{p u i}}{\rho_{0}}=\xi(r)=1-\exp \left[-\Lambda\left(\frac{r}{r_{0}}-1\right)\right]
$$

where the notation: $\Lambda=n_{H \infty} \sigma_{e x} r_{0}$, with $r_{0}=1 \mathrm{AU}$ and $n_{H \infty}=0.05 \mathrm{~cm}^{-3}$ (Izmodenov et al., 1999), has been used. 
Table 1. Estimated latitudinal solar wind speeds $v_{\theta}$ in $[\mathrm{km} / \mathrm{s}]$ as a function of the solar distance $r$ in [AU] along an off-upwind radian with latitude of $\theta \simeq 15^{\circ}$.

\begin{tabular}{lccccc}
\hline$r[\mathrm{AU}]$ & 10 & 20 & 40 & 60 & 80 \\
$v_{\theta}[\mathrm{km} / \mathrm{s}]$ & -2.3 & -6.9 & -16.1 & -25.4 & -35.3 \\
\hline
\end{tabular}

This then helps to evaluate Eq. (7) in the following approximative form:

$$
\begin{aligned}
v_{\theta} & =2 \alpha\left\langle\frac{\partial V_{w}}{\partial \theta}\right\rangle \Lambda\left(\frac{r}{r_{0}}-1\right) \\
& =2 \alpha\left\langle\frac{V_{w f}-V_{w s}}{\Delta \theta}\right\rangle \Lambda\left(\frac{r}{r_{0}}-1\right),
\end{aligned}
$$

where the latitudinal gradient of the solar wind velocity, as it appears during solar minimum conditions (see McComas et al., 2000), has been approximated by a linear change from low speed near-ecliptic wind with a velocity $V_{w s}$, to high speed wind with $V_{w f}$ at higher latitudes with $\theta \geq \Delta \theta$. With the assumptions:

$$
V_{w f}=800 \mathrm{~km} / \mathrm{s} ; \quad \mathrm{V}_{\mathrm{ws}}=400 \mathrm{~km} / \mathrm{s} ; \quad \Delta \theta=30^{\circ},
$$

one then finds the results listed in Table 1 for the upcoming latitudinal solar wind velocity component at increasing distances $r$ from the Sun.

Learning from the above calculated estimates, that a nonnegligible latitudinal velocity component results from latitudinal PUI pressure forces, the need arises to also estimate the density perturbations that the solar wind flow may suffer under these induced non-radial flows. For that purpose we consider briefly the associated form of an approximate mass flow continuity equation.

\section{Approximated continuity equation}

For the radial solar wind flow near the ecliptic one can assume that the latitudinal mass transport into the ecliptic acts as a local source $\Delta \rho / \Delta t$ of additional plasma. Then the simplified mass continuity requires that:

$$
\frac{1}{r^{2}} \frac{d}{d r}\left(r^{2} V_{r}\left(\rho_{0}+\delta \rho\right)\right)=\frac{\Delta \rho}{\Delta t} \simeq 2 \frac{1}{r} \rho_{0} v_{\theta} .
$$

Here, the latitudinal mass flow $\Phi_{\theta}=\rho V_{\theta}$ was presented in its linearized approximation as $\Phi_{\theta}=\rho V_{\theta} \simeq \rho_{0} v_{\theta}$. If, in addition, we make use of

$r^{2} \rho_{0} V_{r}=\mathrm{const}$

and represent for estimation purposes the latitudinal velocity $v_{\theta}$ in the range $r_{0} \leq r \leq r_{\infty}$ by:

$v_{\theta}=v_{\theta \infty}\left(\frac{r}{r_{\infty}}-\frac{r_{0}}{r_{\infty}}\right)$,
Table 2. Estimated relative density variation $\delta \rho / \rho_{0}$ as a function of the solar distance in units of [AU], resulting in the ecliptic due to latitudinal solar wind velocity components.

\begin{tabular}{lcccccc}
\hline$r[\mathrm{AU}]$ & 5 & 10 & 20 & 40 & 60 & 80 \\
$v_{\theta}[\mathrm{km} / \mathrm{s}]$ & 0 & -2.3 & -6.9 & -16.1 & -25.4 & -35.3 \\
$\delta \rho / \rho_{0}$ & 0 & 0.012 & 0.035 & 0.083 & 0.126 & 0.175
\end{tabular}

then we obtain Eq. (10) in the following form:

$\frac{1}{r^{2}} \frac{d}{d r}\left(r^{2} V_{r} \delta \rho\right)=\frac{2}{r} \rho_{00}\left(\frac{r}{r_{0}}\right)^{-2} v_{\theta \infty}\left(\frac{r}{r_{\infty}}-\frac{r_{0}}{r_{\infty}}\right)$

and after integration find:

$\delta \rho(r) \simeq 2 \rho_{0}(r) v_{\theta}(r) / V_{r}$.

The relative density perturbations $\delta \rho / \rho_{0}$ due to the nonradial flows induced by the latitudinal PUI pressure gradient and resulting from Eq. (12) with Eq. (9) is then given in Table 2 .

\section{Towards a more quantitative solution}

In the following we now aim at a more quantitative treatment of the non-radial solar wind flow enforced by the action of latitudinal PUI pressure gradients. For that purpose we start from mass flow and momentum flow conservation requirements formulated by the following set of differential equations written in polar coordinates $r, \theta, \varphi$ :

\subsection{Mass flow continuity equation}

$\frac{1}{r^{2}} \frac{\partial}{\partial r}\left(r^{2} \rho V_{r}\right)+\frac{1}{r \sin \theta} \frac{\partial}{\partial \theta}\left(\sin \theta \rho V_{\theta}\right)=0$

4.2 Momentum flow continuity equations

$$
\begin{aligned}
& \rho V_{r} \frac{\partial V_{r}}{\partial r}+\frac{\rho V_{\theta}}{r} \frac{\partial V_{r}}{\partial \theta}-\rho \frac{V_{\theta}^{2}}{r}=F_{1, r}+F_{2, r} \\
& \rho V_{r} \frac{\partial V_{\theta}}{\partial r}+\frac{\rho V_{\theta}}{r} \frac{\partial V_{\theta}}{\partial \theta}+\rho \frac{V_{r} V_{\theta}}{r}=-\left(\nabla P_{p u i}\right)_{\theta}
\end{aligned}
$$

On the RHS of Eq. (14) the radial forces $F_{1, r}+F_{2, r}$ appear which are due to PUI momentum loading and the radial PUI pressure gradient. According to Eq. (1) these forces are given by:

$F_{r}=F_{1, r}+F_{2, r}=\rho V_{r} \frac{-m_{p} \beta_{e x} \frac{1+\alpha}{\rho_{w}+\rho_{i}}+\frac{2 \alpha}{r} \xi V_{r}}{1+\alpha \xi}$

and with the meaning of $\beta_{\text {ex }}$ given just after Eq. (1) further evaluate to:

$F_{r}=\frac{\rho V_{r}^{2}}{1+\alpha \xi}\left[-(1+\alpha)(1-\xi) \sigma_{e x} n_{H}+\frac{2 \alpha}{r} \xi\right]$. 
Keeping in mind that the PUI abundance $\xi$ at larger distances $(r \geq 30 \mathrm{AU})$ can be approximated by $\xi(r) \simeq \sigma_{\text {ex }} n_{H} r$ (see, e.g. Fahr, 2002) one finds:

$F_{r} \simeq \rho V_{r}^{2} \frac{\left[(\alpha-1) \sigma_{e x} n_{H}\right]}{1+\alpha \sigma_{e x} n_{H} r}$

which, in view of the fact that $1 \gg \alpha \sigma_{e x} n_{H} r$, simply yields:

$F_{r} \simeq(\alpha-1) \sigma_{e x} n_{H} \rho V_{r}^{2}$.

The same approximation can be used to solve Eq. (5), which is used in Eq. (23) below.

In order to arrive at a consistent solution of the system of Eqs. (13), (14) and (15) we now use the following linearization procedure.

\subsection{Linearization}

Setting

$$
\begin{aligned}
\boldsymbol{V} & =V_{r} \boldsymbol{e}_{r}+v_{\theta}(r, \theta) \boldsymbol{e}_{\theta} \\
\rho & =\rho_{0}+\delta \rho \\
V_{r}(r, \theta) & =V_{r 0}(\theta)+v_{r}(r, \theta) \\
v_{r}(r, \theta) & =-V_{r 0}(\theta)(1-\alpha) \sigma_{e x} n_{H \infty}\left(r-r_{0}\right) \\
F_{r} & =(\alpha-1) \sigma_{e x} n_{H} \rho_{0} V_{r 0}^{2},
\end{aligned}
$$

where the expression for $v_{r}(r, \theta)=V_{r 0} \cdot \Delta V_{w}$ was taken from Eq. (5). Neglecting nonlinear terms in the perturbed quantities will then allow us to obtain the following relations. From Eq. (13) for the terms of zeroth order one obtains:

$$
\frac{1}{r^{2}} \frac{\partial}{\partial r}\left(r^{2} \rho_{0} V_{r 0}\right)=0
$$

leading to the following result:

$\Phi_{\theta}(\theta)=r^{2} \rho_{0} V_{r 0}(\theta)$

or equivalently to:

$\rho_{0}(r, \theta)=\Phi_{\theta} /\left(r^{2} V_{r 0}(\theta)\right)$.

Also from Eq. (13) we obtain the following relation for the first order terms

$$
\begin{aligned}
\frac{V_{r 0}(\theta)}{r^{2}} \frac{\partial}{\partial r}\left(r^{2} \delta \rho\right) & +\frac{1}{r^{2}} \frac{\partial}{\partial r}\left(r^{2} \rho_{0} v_{r}\right) \\
& +\frac{\rho_{0}(r)}{r \sin \theta} \frac{\partial}{\partial \theta}\left(\sin \theta v_{\theta}\right)=0 .
\end{aligned}
$$

With the use of Eqs. (5) and (23) one can then transform the above equation into the first first-order relation:

$$
\begin{aligned}
\frac{V_{r 0}(\theta)}{r^{2}} \frac{\partial}{\partial r}\left(r^{2} \delta \rho\right) & +\frac{\rho_{0}(r)}{r \sin \theta} \frac{\partial}{\partial \theta}\left(\sin \theta v_{\theta}\right) \\
& =\rho_{0}(\alpha-1) n_{H_{\infty}} \sigma_{e x} V_{r_{0}}
\end{aligned}
$$

From Eq. (14) the second first-order relation is found:

$\rho_{0} V_{r 0} \frac{\partial v_{r}}{\partial r}+\frac{\rho_{0} v_{\theta}}{r} \frac{\partial V_{r 0}}{\partial \theta}=F_{r}$

and finally, from Eq. (15) the third first-order relation is given by:

$\rho_{0} V_{r 0} \frac{\partial v_{\theta}}{\partial r}+\rho_{0} \frac{V_{r 0} v_{\theta}}{r}=-\left(\nabla P_{p u i}\right)_{\theta}$.

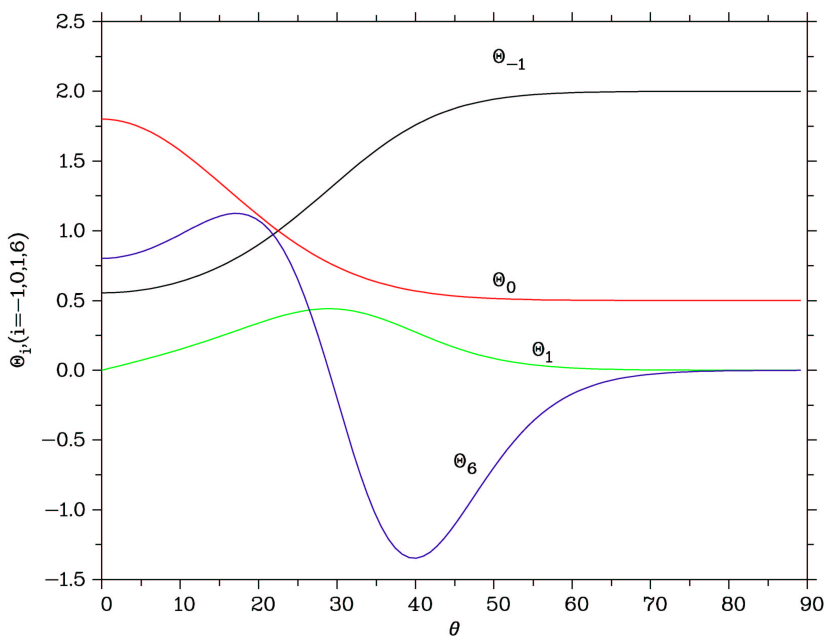

Fig. 1. Shown are the following $\theta$ dependent normalized functions: The black curve $\left(\Theta_{-1}\right)$ represents the latitudinal dependence of the solar wind speed, while the red curve $\left(\Theta_{0}\right)$ represents that of the solar wind number density. The green curve is the function $\Theta_{1}$, and the magenta curve is the function $\Theta_{6}$, both of which are used in the solutions of the differential equations. $\Theta_{1}$ and $\Theta_{6}$ are the first and second derivative with respect to $\theta$ of $\Theta_{-1}=V_{r}(\theta)$.

\section{Latitudinal gradients}

Next, we shall give expressions for the latitudinal gradients of the main solar wind velocity $V_{r 0}(\theta)$ and the pick-up ion pressure $P_{p u i}$. At least under solar minimum conditions, strong systematic, i.e. monotonic variations of the solar wind velocity with latitude, have been recognized (see McComas et al., 2000, 2002). To be based in what follows as close as possible on experimental data we start from ULYSSES plasma measurements published by McComas et al. (2000), representing typical solar minimum conditions in the heliosphere. As obtained from the first full polar orbit passage of ULYSSES, the essential solar wind parameters scaled to a reference distance of $r_{0}=1 \mathrm{AU}$, such as solar wind bulk velocity $V_{r 0}$ and density $\rho_{0}$, have been obtained as functions of solar latitude angle $\theta$.

Concerning the bulk velocity $V_{r 0}(\vartheta)$ one can derive the following analytic representation of these data:

$$
\begin{aligned}
V_{r 0}(\theta)= & V_{r s}+\frac{V_{r f}-V_{r s}}{2} \\
& \cdot\left[1-\tanh \left(\Delta_{\theta}\left(\cos \theta-\cos \theta_{c}\right)\right],\right.
\end{aligned}
$$

where the quantities $V_{r s}=380 \mathrm{~km} / \mathrm{s}$ and $V_{r f}=760 \mathrm{~km} / \mathrm{s}$ are the observed wind speeds for slow and fast solar wind, respectively, and where in our model $\theta_{c}=\pi / 8 \approx 22.5^{\circ}$ and $\Delta_{\theta}=2 \pi$ characterize the critical latitude and the rapidity of the change from slow to fast solar wind (see Fig. 1 for a representation of $V_{r}=\Theta_{-1}$ ).

As a consequence from Eq. (10), the associated solar wind density at the inner boundary is adopted such that the resulting mass flux at $5 \mathrm{AU}$ is constant and equal to $\Phi_{m 0}(5 \mathrm{AU})=v_{s w} \cdot n_{s w}(5 \mathrm{AU}) m_{p}=1.2 \cdot 10^{7} m_{p} \mathrm{~cm}^{-2} \mathrm{~s}^{-1}$, 


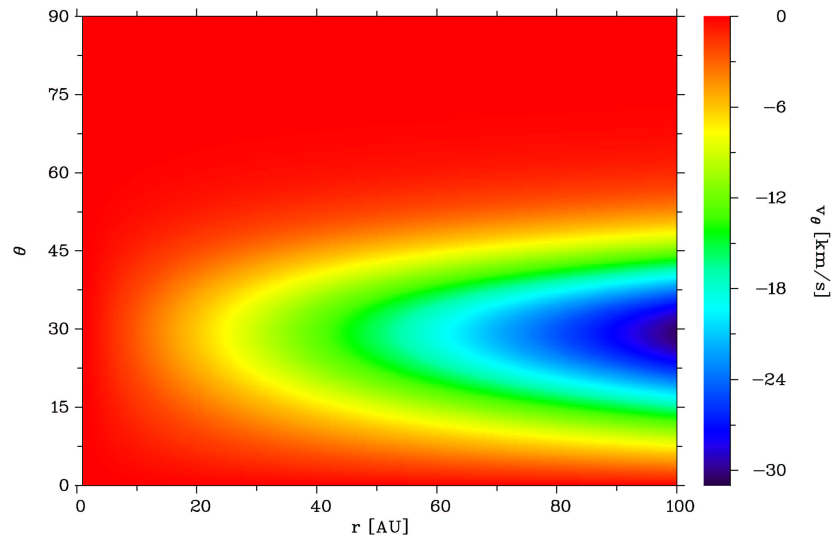

Fig. 2. The latitudinal velocity component. The solar distance is given in $\mathrm{AU}$ ( $x$-axis), the latitude in degrees $(y$-axis), and the speed is colour-coded. For details see text.

which is a value supported by the observations from the ULYSSES spacecraft (McComas et al., 2000). Expressed in a formula this yields:

$\rho_{00}(\theta)=$

$$
\frac{\Phi_{m 0}}{V_{r s}+1 / 2\left(V_{r f}-V_{r s}\right)\left[1-\tanh \left(\Delta_{\theta}\left(\cos \theta-\cos \theta_{c}\right)\right]\right.} .
$$

The quasi-constant behavior of the solar wind mass flow was already recognized in earlier work by Schwenn (1983) and Philips et al. (1996).

Keeping in mind that the pick-up ion pressure $P_{\text {pui }}$ has been represented in the form:

$P_{\text {pui }}=\alpha \rho_{\text {pui }} V_{r 0}^{2}(\theta)$

and that according to Chashei et al. (2003), the density $\rho_{p u i}$ can be given by:

$\rho_{\text {pui }} \simeq \rho_{00}(\theta)\left[1-e^{\left(1-\frac{r}{r_{0}}\right) \Lambda}\right]$

with the notation: $\Lambda=n_{H \infty} \sigma_{e x} r_{0}$, where the LISM H-atom density is denoted by $n_{H \infty}$, the charge exchange cross section for proton - H-atom collisions is denoted by $\sigma_{e x}$, with a reference distance of $10 \mathrm{AU}$ by $r_{0}$. The latitudinal pick-up ion gradient hence is given by:

$$
\begin{aligned}
\frac{\partial}{\partial \theta} P_{p u i} & =\frac{\partial}{\partial \theta} \alpha \rho_{p u i} V_{r 0}^{2}(\theta) \\
& =\alpha\left[1-e^{\left(1-\frac{r}{r_{0}}\right) \Lambda}\right] \frac{\partial}{\partial \theta}\left[\rho_{00}(\theta) V_{r 0}^{2}(\theta)\right] \\
& =\alpha \rho_{00}(\theta) V_{r 0}\left[1-e^{\left(1-\frac{r}{r_{0}}\right) \Lambda}\right] \frac{\partial}{\partial \theta}\left[V_{r 0}(\theta)\right],
\end{aligned}
$$

because $\rho_{00}(\theta) V_{r 0}=\Phi_{m 0}=$ const.

\section{Solution of the differential equations}

As shown in detail in the Appendix, the differential Eqs. (29), (30) and (31) have the solutions:

$$
v_{\theta}=-\alpha \Theta_{1}\left(1-\frac{r_{0}}{r}\left[1+\frac{1}{\Lambda}\left(1-e^{\left(1-\frac{r}{r_{0}}\right) \Lambda}\right)\right]\right)
$$

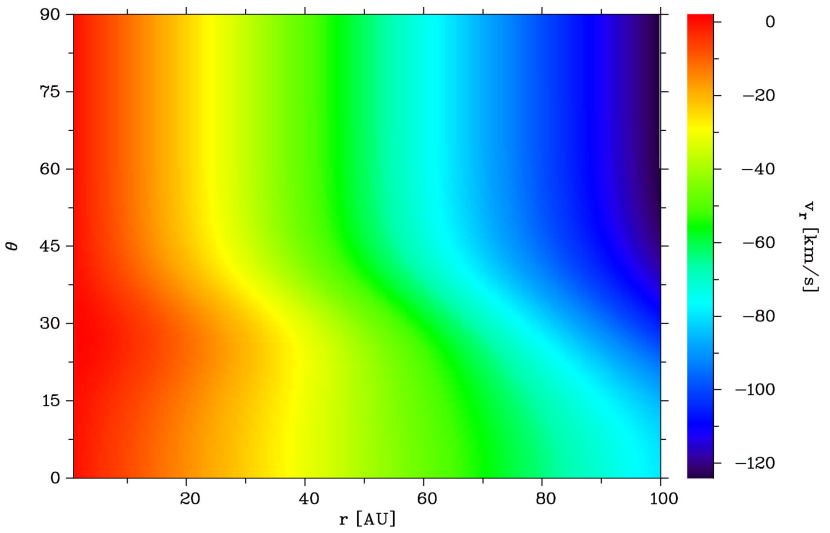

Fig. 3. The perturbation of the radial velocity component. The labels are analogous to that of Fig. 2.

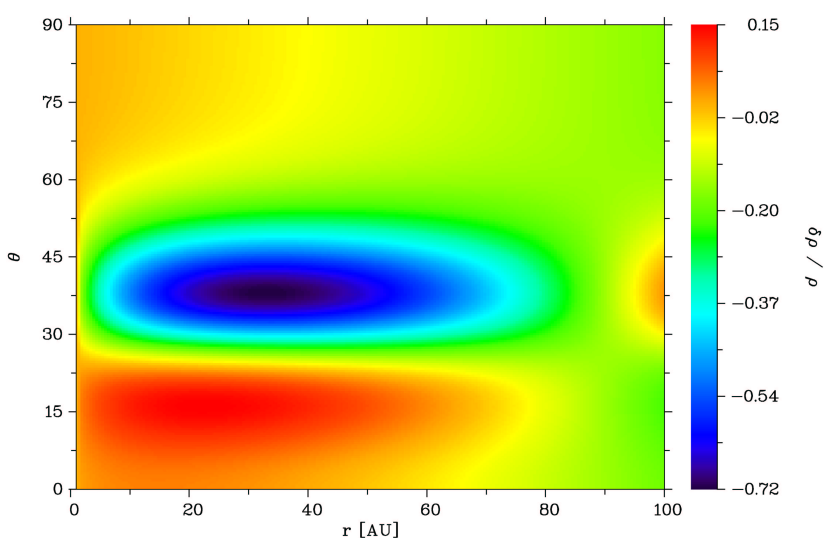

Fig. 4. The ratio of density perturbation and the undisturbed density: $\delta \rho / \rho_{0}$. The labels are analogous to that of Fig. 2, except for the colour-code, which now gives the ratio of the density perturbation and the undisturbed density.

$v_{r}=\Theta_{2}\left(\frac{r}{r_{0}}-1\right)-\Theta_{3} R(r)$
$\delta \rho=\Theta_{10}\left(\frac{r_{0}}{r}-\frac{r_{0}^{2}}{r^{2}}\right)-\Theta_{7} \frac{r_{0}^{2}}{r^{2}} R(r)$

with

$$
\begin{aligned}
R(r) & =1-\frac{r_{0}}{r}-\left(\ln \left(\Lambda \frac{r}{r_{0}}\right)+\frac{r_{0}}{\Lambda r}\right)\left(1-e^{\left(1-\frac{r}{r_{0}}\right) \Lambda}\right) \\
& +e^{\Lambda} \int_{\Lambda}^{\frac{\Lambda r}{r_{0}}} e^{-t} \ln t d t
\end{aligned}
$$

and with the functions $\Theta_{i}$ only dependent on $\theta$ given by:

$$
\begin{aligned}
\Theta_{1} & \sim \sin \theta \cosh ^{-2} \theta^{\prime} \\
\Theta_{3} & \sim \Theta_{1}^{2} / V_{r}(\theta) \\
\Theta_{6} & \sim\left(\cos \theta+2 \Delta_{\theta} \sin ^{2} \theta \tanh \theta^{\prime}\right) \\
\Theta_{7} & \sim\left(\Theta_{1} \cot \theta+\Theta_{6}\right) V_{w}^{-2}
\end{aligned}
$$


while $\Theta_{2}$ and $\Theta_{10}$ are constants. The amplitudes of these functions can be found in the Appendix.

The solutions $v_{r}, v_{\theta}$ and $\delta \rho$ are represented in Figs. 2 to 4. The latitudinal velocity has its largest magnitude at $30^{\circ}$ and large distances, according to the maximum of the function $\Theta_{1}$ (see Fig. 1). The radial velocity (Fig. 3) is decelerated at all latitudes and at large distances. Close to the Sun (e.g. inside $10 \mathrm{AU}$ ) the effect vanishes. In Fig. 4 the ratio of the density variation to the normalized density $\left(\delta \rho / \rho_{0}\right)$ is shown, which is smooth close to the Sun and for small latitudes, reaching its maximum at about $40^{\circ}$ (i.e. for mid latitudes) and decreasing for larger distances and high latitudes.

With the angle $\theta_{c}$ it is easily possible to shift the maxima of the latitudinal velocity and density variation to other latitudinal positions, while the factor $\Delta_{\theta}$ allows one to control the width of the maxima in these functions.

The functions $v_{r}, v_{\theta}$, and $\delta \rho$ are only defined to a distance near the termination shock. In that region the linearization must fail and a full nonlinear description, including the interaction with the termination shock, is needed. On the other hand, the linearized equations are independent of the shock position, and we have set as a limiting distance $100 \mathrm{AU}$. Therefore, the shock position can be estimated independently and close to that distance the above solutions should be appropriate.

\section{The magnetic pressure}

The suggestion of Archimedian magnetic field pressures acting upon the solar wind outflow at large distances and causing meridional flow components was first made by Suess and Nerney (1973), in view of latitudinal pressure gradients which evidently are connected with Parker's spirally wound frozen-in field. In the past the authors had envisioned a spherically symmetric solar wind, while nowadays strong latitudinal solar wind velocity gradients, both at solar minimum and maximum conditions, have been identified with ULYSSES (see McComas et al., 2000, 2003). It may thus be interesting here to briefly revisit this magnetic pressure concept by Suess and Nerney, but now taking into account these latitudinal velocity structures.

The frozen-in Archimedian magnetic field vector $\boldsymbol{B}_{A}$ is given in the following form:

$\boldsymbol{B}_{A}=B_{0}\left(\frac{r_{0}}{r}\right)^{2}\left[\boldsymbol{e}_{r}-\boldsymbol{e}_{\theta} \tan \Psi\right]$,

where $\boldsymbol{e}_{r, \theta}$ denote unit vectors in the $r$ - and in the $\theta$ - directions, and where $\tan \Psi$ is given by:

$\tan \Psi=\frac{\Omega_{s}\left(r-r_{0}\right)}{V_{r}(\theta)} \cos \theta$,

with $\Omega_{s}$ being the angular frequency of the Sun. The magnetic field pressure is now derived from Eqs. (44) and (45).

$$
\begin{aligned}
P_{A} & =B_{A}^{2} / 8 \pi \\
& =B_{A 0}^{2}\left(\frac{r_{0}}{r}\right)^{4}\left[1+\left(\frac{\Omega_{s}\left(r-r_{0}\right)}{V_{r}(\theta)} \cos \theta\right)^{2}\right]
\end{aligned}
$$

which delivers the latitudinal pressure gradient in the following form:

$$
\begin{aligned}
& \frac{\partial P_{A}}{\partial \theta}= B_{A 0}^{2}\left(\frac{r_{0}}{r}\right)^{4} 2\left[1+\frac{\Omega_{s}\left(r-r_{0}\right)}{V_{r}(\theta)} \cos \theta\right] \\
& \cdot \Omega_{S}\left(r-r_{0}\right) \frac{\partial}{\partial \theta}\left(\frac{\cos \theta}{V_{r}}\right) .
\end{aligned}
$$

This pressure gradient should now be compared with the latitudinal PUI pressure gradient which we have taken into account in this paper here:

$\frac{\partial P_{p u i}}{\partial \theta} \simeq \frac{\partial}{\partial \theta}\left(\alpha \rho_{p u i}(r) V_{r}^{2}(\theta)\right)=\frac{2 P_{p u i}}{V_{r}} \frac{\partial V_{r}}{\partial \theta}$.

For regions with $r \gg r_{0}$ near the ecliptic with $\theta \simeq 0$ and $\cos \theta \simeq 1$ one then finds:

$\delta P=\frac{-\frac{\partial P_{A}}{\partial \theta}}{\frac{\partial P_{p u i}}{\partial \theta}} \simeq \frac{2 \frac{P_{A}}{V_{r}} \frac{\partial V_{r}}{\partial \theta}}{\frac{2 P_{p u i}}{V_{r}} \frac{\partial V_{r}}{\partial \theta}}=\frac{P_{A}}{P_{p u i}}=\left(2 \alpha \xi M_{A}^{2}\right)^{-1}$,

where $M_{A}$ denotes the Alfvén Mach number of the solar wind flow and $\xi$ is again the relative abundance of pick-up ions with respect to solar wind protons.

Inserting now concrete numbers for the above quantities at $r \geq 20 \mathrm{AU}$ one may be convinced that $\delta P \leq(1 / 20)$. This expresses the fact that latitudinal magnetic pressures, though acting opposite to the pick-up ion pressures, do not change the results presented in the section before.

\section{Discussion and concluding remarks}

In the preceding sections of this paper we have developed a theoretical description of the three-dimensional solar wind outflow under the action of radial and latitudinal pick-up ion pressure gradients. In this paper we started out from the typical solar wind flow configuration which was found for typical solar minimum conditions during the first and second polar latitude passage of the ULYSSES space probe (see McComas et al., 2000). As a consequence of the strong latitude gradients in the radial solar wind velocity and density, associated latitudinal pick-up ion pressure gradients can be derived which induce nonnegligible latitudinal solar wind velocity components. As a consequence of the induced non-radial solar wind flow configurations, complicated structures of perturbed solar wind density and velocity components arise.

In Figs. 2 through 4 iso-contours of these perturbations are shown, which can be taken as a visual aid for the interpretation of plasma measurements of distant heliospheric space probes, such as VOYAGER-1/2 or PIONEER-10/11 (see, e.g. Whang, 1998; Wang et al., 2000; Richardson et al., 2003). In Fig. 2 one can see the 2-dimensional distribution of latitudinal solar wind speeds as a function of solar distance $r$ in [AU] and of latitude $\theta$. At small distance $r<10$ AU nearly no latitudinal velocity is found. Also at ecliptic latitudes $\theta<7^{\circ}$ and at polar latitudes $\theta>50^{\circ}$ no latitudinal velocities are developing, even up to large distances. At intermediate latitudes $10^{\circ}<\theta<45^{\circ}$, however, astonishingly pronounced 
latitudinal velocities, always directed towards the ecliptic, with magnitudes of up to $30 \mathrm{~km} / \mathrm{s}$ are established. Especially at large distances and latitudes of around $\theta=30^{\circ}$ relatively large latitudinal velocities are predicted by our theoretical calculations. The latter fact should induce even stronger flow perturbations in the near-ecliptic region downstream of the termination shock, since here latitudinal components are conserved while radial components are strongly reduced.

In Fig. 3 the resulting perturbations in the radial solar wind velocities is shown. As evident in all cases decelerations of the original radial solar wind velocity occur with increasing magnitudes, resulting both at larger distances and at larger latitudes. Concerning the latter fact one should bear in mind that absolute radial velocity perturbations are shown in this figure. In view of the latitude profile of the original radial solar wind velocity it is thus found that the relative decelerations of the radial solar wind velocity are larger (i.e. resulting to about 20\%) at small latitudes compared to those resulting at larger latitudes. This is also partially explaining the resulting density perturbations, which are shown in Fig. 4.

In this figure a colour-coded $r-\theta$ distribution of the resulting relative density perturbations connected with the perturbed solar wind flow is shown, clearly depicting separated regions where density reductions and density enhancements occur. Density enhancements hereby result from two reasons. One is the fact that in a decelerated solar wind flow, density is falling off with distance less than by $\left(r^{-2}\right)$. The other one, operating in regions near the ecliptic, is due to the fact that connected with latitudinal wind velocities solar wind plasma is transported to lower latitudes due to converging flows. In the region between latitudes $25^{\circ}<\theta<45^{\circ}$ one can notice relative density reductions of up to $30 \%$, which are due to latitudinal plasma transports.

All the colour-contour plots shown in Figs. 2 through 4 depict solar wind structures which are expected for typical solar wind minimum conditions, when the original solar wind outflow $(r<5 \mathrm{AU})$ is characterized by the strongly pronounced velocity pattern with low speed at low latitudes and fast speed at larger ones (see McComas et al., 2000). During the evolution of the solar cycle from minimum towards solar maximum, this basic feature changes and will become much more complicated and sub-structured, developing a changing number of high-velocity fingers superimposed on low velocity winds distributed over all latitudes (see McComas et al., 2003). This latter velocity pattern was not taken into account in a quantitative form in this paper. Qualitatively, one can, however, predict from the experiences made here for the solar minimum conditions that the action of pick-up ion pressure gradients clearly will smooth the latitude structures of fastand low-speed features; hence, at larger distances the original latitude structure feature of the the solar wind velocity pattern will slowly fade off.

In Fig. 5 the dependence of the solar wind deceleration on the interstellar hydrogen density $n_{H_{\infty}}$ is shown. As we have described in the beginning of this paper, the reduction of the radial solar wind velocity not only is caused by pick-up ion momentum loading of the primary solar wind, but also by

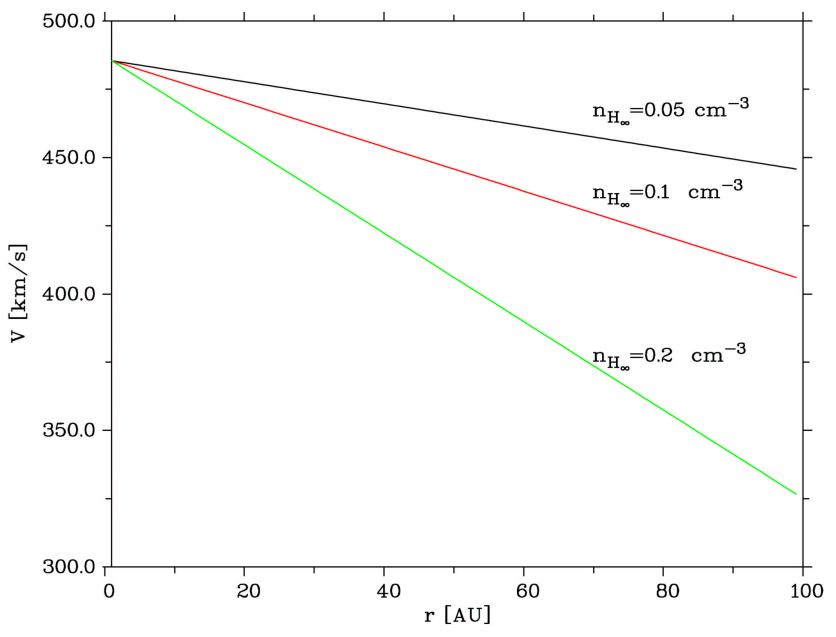

Fig. 5. The dependence of the radial solar wind speed deceleration on the atomic hydrogen density in the interstellar medium. The solar wind speed ( $y$-axis) is the sum of the undisturbed bulk velocity of the solar wind $V_{w}$ and the perturbation $v_{r}$ (see Fig. 3). The solar wind speed decreases is shown for three different $\mathrm{H}$-atom number densities, e.g. $n_{H_{\infty}}=0.05,0.1,0.2 \mathrm{~cm}^{-3}$ represented by the black, red and green curve, respectively.

the action of the pick-up ion pressure gradient, which partly compensates the momentum loading effect. In Fig. 5 for the latitude $\theta=+30^{\circ}$, at which approximately VOYAGER-1 is presently flying, the expected solar wind velocity profile at solar minimum conditions is shown, calculated for various interstellar hydrogen densities. As clearly can be seen in the figure, decelerations by about $10 \%$ at distances of $60 \mathrm{AU}$, as found by Wang et al. (2000), are obtained with interstellar gas densities of about $n_{H_{\infty}}=0.1 \mathrm{~cm}^{-3}$ or even with slightly larger values. Without taking into account the pick-up ion pressure gradient effect Wang et al. (2000) had claimed for a density of only $n_{H_{\infty}}=0.05 \mathrm{~cm}^{-3}$ which, as was shown in this paper, is too low by a factor of 2 . This means that higher interstellar gas densities are thus conciliatory with the solar wind decelerations seen by VOYAGER-1.

As was shown in the previous section, the magnetic pressure is neglible compared to the latitudinal PUi pressure in the outer heliosphere.

Finally, the consequence of latitudinal velocities for the shocked solar wind are discussed. When at latitudes $\theta= \pm 30^{\circ}$ near the upwind direction the solar wind arrives at the termination shock it has developed latitudinal velocity components of the order of $30 \mathrm{~km} / \mathrm{s}$. Since the shock surface here is likely to have a shock normal close to the radial orientation, one can easily deduce that radial velocity components are reduced by about a factor of $1 / 4$ (i.e. strong shock), whereas the latitudinal components are conserved at the shock transition. This means that the subsonic flow in the direction downstream of the shock has a strong tilt by about 20 degrees with respect to the shock normal. This means, however, that beyond the termination shock a subsonic flow pattern with pronounced convergence towards the upwind 
axis is induced and, as a consequence, the downstream plasma density should be increased on the upwind axis between the termination shock and the heliopause.

\section{Appendix A}

First, we collect all variables and define some shorthand notations by:

$$
\begin{aligned}
\theta^{\prime}= & \left\{\Delta_{\theta}\left(\cos \theta-\cos \theta_{c}\right)\right\} \\
\frac{\partial \theta^{\prime}}{\partial \theta} & =-\Delta_{\theta} \sin \theta \\
\rho_{0}= & \rho_{00} \frac{r_{0}^{2}}{r^{2}} \\
V_{r}= & V_{w}(\theta) \\
= & V_{r s}+\frac{V_{r f}-V_{r s}}{2}\left[1-\tanh \left\{\Delta_{\theta}\left(\cos \theta-\cos \theta_{c}\right)\right\}\right] \\
= & V_{r s}+\frac{V_{r f}-V_{r s}}{2}\left[1-\tanh \theta^{\prime}\right] \\
\Theta_{1}= & \frac{\partial V_{r}}{\partial \theta}=\Delta_{\theta} \frac{V_{r f}-V_{r s}}{2} \frac{\sin \theta}{\cosh ^{2} \theta^{\prime}} \\
\rho_{\text {pui }}= & \rho_{0}\left(1-e^{\left(1-\frac{r}{r_{0}}\right) \Lambda}\right) \\
& =\rho_{00} \frac{r_{0}^{2}}{r^{2}}\left(1-e^{\left(1-\frac{r}{r_{0}}\right) \Lambda}\right) \\
P_{\text {pui }}= & \alpha \rho_{\text {pui }} V_{w}^{2},
\end{aligned}
$$

where $V_{r s}, V_{r f}, \alpha_{c}$, and $\theta_{c}$ are constants. Their values are discussed in the text. The gradient of the PUI is given by:

$$
\begin{aligned}
&-\nabla P_{p u i}=-\left(\frac{\partial}{\partial r}, \frac{1}{r} \frac{\partial}{\partial \theta}\right) \alpha \rho_{p u i} V_{w}^{2} \\
&-\left.\nabla P_{p u i}\right|_{\theta}=- \frac{\alpha}{r} \frac{r_{0}^{2}}{r^{2}}\left(1-e^{\left(1-\frac{r}{r_{0}}\right) \Lambda}\right) \\
& \cdot \rho_{00} V_{w} \Delta_{\theta} \frac{V_{r f}-V_{r s}}{2} \frac{\sin \theta}{\cosh ^{2} \theta^{\prime}} .
\end{aligned}
$$

The last differential Eq. (31) reads:

$$
\begin{aligned}
\rho_{0} V_{w} \frac{\partial v_{\theta}}{\partial r} & +\rho_{0} V_{w} \frac{v_{\theta}}{r} \\
& =-\frac{\alpha \rho_{00} V_{w}}{r} \frac{r_{0}^{2}}{r^{2}}\left(1-e^{\left(1-\frac{r}{r_{0}}\right) \Lambda}\right) \Theta_{1} \\
& =-\frac{\alpha}{r} \rho_{0} V_{w}\left(1-e^{\left(1-\frac{r}{r_{0}}\right) \Lambda}\right) \Theta_{1}
\end{aligned}
$$

where $\Theta_{1}$ is independent of $r$.

Then Eq. (A10) can be written in the form:

$$
\frac{\partial v_{\theta}}{\partial r}+\frac{v_{\theta}}{r}=-\frac{\alpha \Theta_{1}}{r}\left(1-e^{\left(1-\frac{r}{r_{0}}\right) \Lambda}\right)
$$

with the solution:

$$
\begin{aligned}
v_{\theta}= & \frac{r_{0}}{r} v_{\theta, 0}-\alpha \Theta_{1}\left(1-\frac{r_{0}}{r}\right) \\
& +\alpha \frac{r_{0}}{r} \frac{\Theta_{1}}{\Lambda}\left(1-e^{\left(1-\frac{r}{r_{0}}\right) \Lambda}\right),
\end{aligned}
$$

where $v_{\theta, 0}$ is an integration constant which can be set equal to zero because at $r=r_{0}$ no latitudinal wind component is admitted.

The second differential Eq. (30) is:

$\rho_{0} V_{w} \frac{\partial v_{r}}{\partial r}+\frac{\rho_{0} v_{\theta}}{r} \frac{\partial V_{w}}{\partial \theta}=F_{r}=(\alpha-1) \sigma_{e x} n_{H} \rho_{0} V_{w}^{2}$

and can be written as follows:

$$
\begin{aligned}
\frac{\partial v_{r}}{\partial r}+\frac{\Theta_{1}}{V_{w}}\left[\frac{r_{0}}{r^{2}} v_{\theta, 0}-\frac{\alpha \Theta_{1}}{r}\left(1-\frac{r_{0}}{r}\right)\right. \\
\left.+\frac{\alpha r_{0} \Theta_{1}}{r^{2} \Lambda}\left(1-e^{\left(1-\frac{r}{r_{0}}\right) \Lambda}\right)\right]=\frac{\Theta_{2}}{r_{0}}
\end{aligned}
$$

or

$\frac{\partial v_{r}}{\partial r}=\Theta_{2} \frac{1}{r_{0}}+\Theta_{3} \frac{1}{r}+\Theta_{4} \frac{r_{0}}{r^{2}}+\Theta_{5} \frac{r_{0}}{r^{2}} e^{\left(1-\frac{r}{r_{0}}\right) \Lambda}$

with

$$
\begin{aligned}
\Theta_{2} & =(\alpha-1) \sigma_{e x} n_{H} V_{w} r_{0}=(\alpha-1) \Lambda V_{w} \\
\Theta_{3} & =-\frac{\alpha \Theta_{1}^{2}}{V_{w}} \\
\Theta_{4} & =\frac{\Theta_{1}}{V_{w}}\left[v_{\theta, 0}+\alpha \Theta_{1}\left(1+\frac{1}{\Lambda}\right)\right] \\
\Theta_{5} & =-\frac{\alpha \Theta_{1}^{2}}{V_{w} \Lambda}
\end{aligned}
$$

and has the solution:

$$
\begin{aligned}
v_{r} & =v_{r_{0}}-\Theta_{2}\left(1-\frac{r}{r_{0}}\right)+\Theta_{3} \ln \left(\frac{r}{r_{0}}\right)-\Theta_{4}\left(\frac{r_{0}}{r}-1\right)+\Theta_{5} r_{0} \int_{r_{0}}^{r} \frac{e^{\left(1-\frac{r}{r_{0}}\right) \Lambda}}{r^{2}} d r \\
& =v_{r_{0}}-\Theta_{2}\left(1-\frac{r}{r_{0}}\right)+\Theta_{3} \ln \left(\frac{r}{r_{0}}\right)-\Theta_{4}\left(\frac{r_{0}}{r}-1\right)+\left.\Theta_{5} r_{0} e^{\Lambda}\left(-\frac{e^{-\Lambda \frac{r}{r_{0}}}}{r}-\frac{\Lambda}{r_{0}} \operatorname{Ei}\left(-\Lambda \frac{r}{r_{0}}\right)\right)\right|_{r_{0}} ^{r} \\
& =v_{r_{0}}-\Theta_{2}\left(1-\frac{r}{r_{0}}\right)+\Theta_{3} \ln \left(\frac{r}{r_{0}}\right)-\Theta_{4}\left(\frac{r_{0}}{r}-1\right)
\end{aligned}
$$




$$
+\Theta_{5}\left(1-\frac{r_{0}}{r} e^{\left(1-\frac{r}{r_{0}}\right) \Lambda}\right)-\Theta_{5} \Lambda\left[\ln \left(\Lambda \frac{r}{r_{0}}\right) e^{\left(1-\frac{r}{r_{0}}\right) \Lambda}-\ln \Lambda\right]-\Theta_{5} \Lambda e^{\Lambda} \int_{\Lambda}^{\frac{\Lambda r}{r_{0}}} e^{-t} \ln t d t,
$$

where $v_{r_{0}}$ is an integration constant. The first differential Eq. (28) reads:

$\frac{V_{w}}{r^{2}} \frac{\partial}{\partial r}\left(r^{2} \delta \rho\right)+\rho_{00} \frac{r_{0}^{2}}{r^{3}} \frac{1}{\sin \theta}\left[v_{\theta} \cos \theta+\sin \theta\left\{-\alpha\left(1-\frac{r_{0}}{r}\right)+\frac{\alpha}{\Lambda} \frac{r_{0}}{r}\left(1-e^{\left(1-\frac{r}{r_{0}}\right)}\right)\right\} \frac{\partial \Theta_{1}}{\partial \theta}\right]=\rho_{00} \frac{r_{0}^{2}}{r^{2}}(\alpha-1) n_{H_{\infty}} \sigma_{e x} V_{r_{0}}$.

The function $\Theta_{1}$ is independent of $r$ and its derivative with respect to $\theta$ yields:

$\Theta_{6}=\frac{\partial \Theta_{1}}{\partial \theta}=\frac{\partial}{\partial \theta}\left(\Delta_{\theta} \frac{V_{r f}-V_{r s}}{2} \frac{\sin \theta}{\cosh ^{2} \theta^{\prime}}\right)=\Delta_{\theta} \frac{V_{r f}-V_{r s}}{2}\left(\frac{\cos \theta}{\cosh ^{2} \theta^{\prime}}+2 \Delta_{\theta} \frac{\sin ^{2} \theta}{\cosh ^{2} \theta^{\prime}} \tanh \theta^{\prime}\right)$. ing:

Inserting the solution for $v_{\theta}$ from Eq. (A12) and introduc-

$$
\begin{aligned}
& \Theta_{7}=-\frac{\rho_{00}}{V_{w}} \alpha\left[\Theta_{1} \cot \theta+\Theta_{6}\right] \\
& \Theta_{8}=\frac{\rho_{00}}{V_{w}}\left[\left\{v_{\theta, 0}+\alpha \Theta_{1}\left(1+\frac{1}{\Lambda}\right)\right\}\right. \\
& \left.\cot \theta+\alpha \Theta_{6}\left\{1+\frac{1}{\Lambda}\right\}\right]
\end{aligned}
$$

$$
\begin{aligned}
& \Theta_{9}=-\frac{\rho_{00}}{V_{w}} \frac{\alpha}{\Lambda}\left[\Theta_{1} \cot \theta+\Theta_{6}\right] \\
& \Theta_{10}=\rho_{00}(\alpha-1) n_{H_{\infty}} \sigma_{e x} r_{0}=\rho_{00}(\alpha-1) \Lambda
\end{aligned}
$$

the differential equation can, after some algebra, be written as:

$$
\frac{\partial}{\partial r}\left(r^{2} \delta \rho\right)=\Theta_{10} r_{0}-\Theta_{7} \frac{r_{0}^{2}}{r}-\Theta_{8} \frac{r_{0}^{3}}{r^{2}}-\Theta_{9} \frac{r_{0}^{3}}{r^{2}} e^{\left(1-\frac{r}{r_{0}}\right) \Lambda} .
$$

Solving for $\delta \rho$ yields:

$$
\begin{aligned}
\delta \rho=\delta \rho_{0} \frac{r_{0}^{2}}{r^{2}}+\Theta_{10}\left(\frac{r_{0}}{r}-\frac{r_{0}^{2}}{r^{2}}\right)-\Theta_{7} \frac{r_{0}^{2}}{r^{2}} \ln \frac{r}{r_{0}}+\Theta_{8}\left(\frac{r_{0}^{3}}{r^{3}}-\frac{r_{0}^{2}}{r^{2}}\right)-\Theta_{9} \frac{r_{0}^{2}}{r^{2}}\left(1-\frac{r_{0}}{r} e^{\left(1-\frac{r}{r_{0}}\right) \Lambda}\right) & \\
& +\Theta_{9} \Lambda \frac{r_{0}^{2}}{r^{2}}\left[\ln \left(\Lambda \frac{r}{r_{0}}\right) e^{\left(1-\frac{r}{r_{0}}\right) \Lambda}-\ln \Lambda-e^{\Lambda} \int_{\Lambda}^{\frac{\Lambda r}{r_{0}}} e^{-t} \ln t d t\right] .
\end{aligned}
$$

All three functions $\left(v_{r}, v_{\theta}\right.$, and $\left.\delta \rho\right)$ are equal to their respective integration constants $v_{r_{0}}, v_{\theta_{0}}$, and $\delta \rho_{0}$ at $\theta=0$. For physical reason, these boundary values should be zero, because the perturbation should vanish at $r=r_{0}$ and therefore, one obtains: $v_{\theta, 0}=v_{r_{0}}=\delta \rho_{0}=0$.

After replacing $\Theta_{4}$ and $\Theta_{5}$ by $\Theta_{3}$ and $\Theta_{8}$ and $\Theta_{9}$ by $\Theta_{7}$ and rearranging, all three solutions are:

$v_{\theta}=-\alpha \Theta_{1}\left(1-\frac{r_{0}}{r}\left[1+\frac{1}{\Lambda}\left(1-e^{\left(1-\frac{r}{r_{0}}\right) \Lambda}\right)\right]\right)$

$v_{r}=\Theta_{2}\left(\frac{r}{r_{0}}-1\right)-\Theta_{3}\left[1-\frac{r_{0}}{r}-\left(\ln \left(\Lambda \frac{r}{r_{0}}\right)+\frac{r_{0}}{\Lambda r}\right)\left(1-e^{\left(1-\frac{r}{r_{0}}\right) \Lambda}\right)+e^{\Lambda} \int_{\Lambda}^{\frac{\Lambda r}{r_{0}}} e^{-t} \ln t d t\right]$

$\delta \rho=\Theta_{10}\left(\frac{r_{0}}{r}-\frac{r_{0}^{2}}{r^{2}}\right)-\Theta_{7} \frac{r_{0}^{2}}{r^{2}}\left[1-\frac{r_{0}}{r}-\left(\ln \left(\Lambda \frac{r}{r_{0}}\right)+\frac{r_{0}}{\Lambda r}\right)\left(1-e^{\left(1-\frac{r}{r_{0}}\right) \Lambda}\right)+e^{\Lambda} \int_{\Lambda}^{\frac{\Lambda r}{r_{0}}} e^{-t} \ln t d t\right]$.

For $r \rightarrow \infty$ the solutions for $v_{\theta}$ reach a constant value, while that for $v_{r} \propto r$ goes linearly to infinity, that of $\delta \rho$ vanishes:

$$
\begin{aligned}
& v_{\theta}(r \rightarrow \infty)=\alpha \Theta_{1} \\
& v_{r}(r \rightarrow \infty) \propto \Theta_{2} r \\
& \delta \rho(r \rightarrow \infty)=0 .
\end{aligned}
$$

On the other hand, the functions $v_{r}, v_{\theta}$, and $\delta \rho$ are only defined to some distance close to and upstream of the termination shock (for a more detailed discussion, see Sect. 5). 
Acknowledgements. The authors are grateful for financial support granted to them by the Deutsche Forschungsgemeinschaft in the frame of the project "Heliotrigger" (Fa 97/28-1).

Topical Editor R. Forsyth thanks R. Kallenbach and another referee for their help in evaluating this paper.

\section{References}

Chalov, S. V. and Fahr, H. J.: Entropy generation at the multi-fluid solar wind termination shock producing anomalous cosmic ray particles, Planet. Space Sci., 43, 1035-1043, 1995.

Chalov, S. V. and Fahr, H. J.: The three-fluid structure of the particle modulated solar wind termination shock., Astron. Astrophys. 326, 860-869, 1997.

Chalov, S. V., Fahr, H. J., and Izmodenov, V.: Spectra of energized pick-up ions upstream of the heliospheric termination shock I. The role of Alfvénic turbulences, Astron. Astrophys., 304, 609616, 1995

Chalov, S. V., Fahr, H. J., and Izmodenov, V.: Spectra of energized pick-up ions upstream of the two-dimensional heliospheric termination shock. II. Acceleration by Alfvénic and by large-scale solar wind turbulences, Astron. Astrophys., 320, 659-671, 1997.

Chashei, I. V., Fahr, H. J., and Lay, G.: Consistent thermodynamics of the MHD wave-heating two-fluid solar wind, Annales Geophysicae, 21, 1-14, 2003.

Fahr, H. J.: Non-Thermal Solar Wind Heating by Supra-Thermal Ions, Solar Physics, 30, 193-218, 1973.

Fahr, H. J.: Global Energy Transfer from Pick-up Ions to Solar Wind Protons, Solar Physics, 208, 335-344, 2002.

Fahr, H. J. and Chahshei, I. V.: On the thermodynamics of MHD wave-heated solar wind protons, Astron. Astrophys., 395, 9911000, 2002.

Fahr, H. J. and Fichtner, H.: The influence of pick-up ion-induced wave pressures on the dynamics of the mass-loaded solar wind, Solar Physics, 158, 353-363, 1995.

Fahr, H. J. and Lay, G.: Remote diagnostic of the heliospheric termination shock using neutralized post shock pick-up ions as messengers, Astron. Astrophys., 356, 327-334, 2000.

Fahr, H. J. and Ripken, H. W.: The physics of the heliospheric interface and its implications for LISM diagnostics, Astron. Astrophys., 139, 551-554, 1984.

Fahr, H. J. and Rucinski, D.: eutral interstellar gas atoms reducing the solar wind Mach number and fractionally neutralizing the solar wind, Astron. Astrophys., 350, 1071-1078, 1999.

Fahr, H. J. and Rucinski, D.: Modification of Properties and Dynamics of Distant Solar Wind Due to its Interaction with Neutral Interstellar Gas, Space Sci.Rev., 97, 407-412, 2001.

Fichtner, H., Le Roux, J. A., Mall, U., and Rucinski, D.: On the transport of pick-up ions in the heliosphere, Astron. Astrophys. 314, 650-662, 1997.

Holzer, T. E. and Leer, E.: Solar Wind Heating Beyond 1 AU, Astropys. Space Sci., 24, 335-347, 1973.

Izmodenov, V. V., Geiss, J., Lallement, R., Gloeckler, G., Baranov, V. B., and Malama, Y. G.: Filtration of interstellar hydrogen in the two-shock heliospheric interface: Inferences on the local interstellar cloud electron density, J. Geophys. Res., 104, 47314742, 1999.
Lee, M. A.: Effects of cosmic rays and interstellar gas on the dynamics of a wind, in: Stellar Winds, edited by Jokipii, J. R., Sonett, C. P., and Giampapa, M., Tucson, Arizona, 857-886, 1997.

McComas, D. J., Barraclough, B. L., Funsten, H. O., Gosling, J. T., Santiago-Muñoz, E., Skoug, R. M., Goldstein, B. E., Neugebauer, M., Riley, P., and Balogh, A.: Solar wind observations over Ulysses' first full polar orbit, J. Geophsy. Res., 105, 10419-10 433, 2000

McComas, D. J., Elliott, H. A., Gosling, J. T., Reisenfeld, D. B., Skoug, R. M., Goldstein, B. E., Neugebauer, M., and Balogh, A.: Ulysses' second fast-latitude scan: Complexity near solar maximum and the reformation of polar coronal holes, Geophys. Res. Lett., 29, 9, doi:0.1029/2001GL014164, 2002.

McComas, D., Elliott, H. A., Schwadron, N. A., Gosling, J. T., Skoug, R. M., and Godlsetin, B. E.: The three-dimensional solar wind around solar maximum, Geophys. Res. Lett., 30, 24-1, 2003, doi:10.1023/2003GL017136.

McNutt, R. L., Lyon, J., and Goodrich, C. C.: Simulation of the heliosphere: Generalized charge-exchange cross sections, J. Geophys. Res., 104, 14 803-14 810, 1999.

Philips, J. L., Bame, S. J., Feldman, W. C., Gosling, J. T., and McComas, D. J.: ULYSSES solar wind plasma observations from peak southerly latitudes through perihelion and beyond, in: SOLAR WIND VIII, Dana Point/CA, edited by Winterhalter, D., Gosling, J. T., Habbal, S. R., Kurth, W. S., and Neugebauer, M., AIP CP-382, 416-423, 1996.

Richardson, J. D., Paularena, K. I., Lazarus, A. J., and Belcher, J. W.: Evidence for a solar wind slowdown in the outer heliosphere?, Geophys. Res. Lett., 22, 1469-1472, 1995.

Richardson, J. D., Wang, C., and Burlaga, L. F.: The solar wind in the outer heliosphere, Adv. Spa. Sci., in press, 2003.

Ripken, H. W. and Fahr, H. J.: Modification of the local interstellar gas properties in the heliospheric interface, Astron. Astrophys., 122, 181-192, 1983.

Schwenn, R.: The "average" solar wind in the inner heliosphere: Structures and slow variations, in SOLAR WIND V, Woodstock/Vermont, edited by Neugebauer, M., NASA CP-2280, 489-496, 1983.

Suess, S. T. and Nerney, S. F.: Meridional Flow and the Validity of the Two-Dimensional Approximation in Stellar-Wind Modeling, Astrophys. J., 184, 17-25, 1973.

Vasyliunas, V. M. and Siscoe, G. L.: On the flux and the energy spectrum of interstellar ions in the solar system, J. Geophys. Res., 81, 1247-1252, 1976.

Wang, C. and Belcher, J. W.: The heliospheric boundary response to large-scale solar wind fluctuations: A gasdynamic model with pick-up ions, J. Geophys. Res., 104, 549-556, 1999.

Wang, C., Richardson, J. D., and Gosling, J. T.: Slowdown of the Solar Wind in the Outer Heliosphere and the Interstellar Neutral Hydrogen Density, Geophys. Res. Lett., 27, 2429-2432, 2000.

Whang, Y. C.: Solar wind in the distant heliosphere, J. Geophys. Res., 103, 17 419-17 428, 1998.

Wu, F. M. and Judge, D. L.: Temperature and flow velocity of the interplanetary gases along solar radii, Astrophys. J., 231, 594$605,1979$. 